\title{
ON THE 2-CATEGORIES OF WEAK DISTRIBUTIVE LAWS
}

\author{
GABRIELLA BÖHM, STEPHEN LACK, AND ROSS STREET \\ Dedicated to Mia Cohen on the occasion of her retirement
}

\begin{abstract}
A weak mixed distributive law (also called weak entwining structure [8) in a 2-category consists of a monad and a comonad, together with a 2-cell relating them in a way which generalizes a mixed distributive law due to Beck. We show that a weak mixed distributive law can be described as a compatible pair of a monad and a comonad, in 2-categories extending, respectively, the 2-category of comonads and the 2-category of monads in 13. Based on this observation, we define a 2-category whose 0 -cells are weak mixed distributive laws. In a 2-category $\mathcal{K}$ which admits Eilenberg-Moore constructions both for monads and comonads, and in which idempotent 2-cells split, we construct a fully faithful 2-functor from this 2 -category of weak mixed distributive laws to $\mathcal{K}^{2 \times 2}$.
\end{abstract}

\section{INTRODUCTION}

Distributive laws - between two monads; between two comonads; or between a monad and a comonad in any bicategory (the latter known as the 'mixed' case) - were discussed by Beck in [1. In Hopf algebra theory the mixed case was introduced in [6] by Brzeziński and Majid in the particular bicategory Bim of Algebras; Bimodules; Bimodule Maps, under the name 'entwining structure', as a tool for unifying various Hopf type modules.

For any 2-category $\mathcal{K}$, there is a 2-category $\operatorname{Mnd}(\mathcal{K})$ of monads in $\mathcal{K}$ for which a monad in $\operatorname{Mnd}(\mathcal{K})$ is the same thing as two monads in $\mathcal{K}$ with a distributive law between them [13. Similarly, a comonad in $\operatorname{Mnd}(\mathcal{K})$ is the same thing as a mixed distributive law. Dually, there is a 2-category $\operatorname{Cmd}(\mathcal{K})=\operatorname{Mnd}\left(\mathcal{K}_{*}\right)_{*}$, where $(-)_{*}$ denotes the vertical opposite of a 2-category (the superscript "co" is also often used). A monad in $\operatorname{Cmd}(\mathcal{K})$ is once again a mixed distributive law, while a comonad in $\operatorname{Cmd}(\mathcal{K})$ is the same as two comonads with a distributive law between them.

We identify the isomorphic 2-categories $\operatorname{Mnd}(\operatorname{Cmd}(\mathcal{K}))$ and $\operatorname{Cmd}(\operatorname{Mnd}(\mathcal{K}))$, and write each as $\operatorname{Mdl}(\mathcal{K})$, the 2-category of mixed distributive laws. A typical object will be written as $(K, t, c, \lambda)$, where $K$ is the underlying object, $t$ the monad, $c$ the comonad, and $\lambda: t c \rightarrow c t$ the 2-cell between them giving the distributive law. In the case where $t$ has a right adjoint $d$, the monad structure on $t$ induces a comonad structure on $d$, and mixed distributive laws $t c \rightarrow c t$ are in bijection with distributive laws $c d \rightarrow d c$.

Distributive laws play a key role in the description of liftings of monads and comonads [13], 12]. If the 2-category $\mathcal{K}$ admits Eilenberg-Moore constructions for monads; that is, the fully faithful inclusion 2-functor $I: \mathcal{K} \rightarrow \operatorname{Mnd}(\mathcal{K})$ possesses a right 2adjoint alg, then there is a fully faithful 2-functor from $\operatorname{Mnd}(\mathcal{K})$ to the arrow 2-category $\mathcal{K}^{2}$ (i.e. the 2-category of 2 -functors from the interval 2-category $0 \rightarrow 1$ to $\mathcal{K}$ ). It

Date: Sep 2010. 
sends a monad $(K, t)$ to the forgetful map $u^{t}: K^{t} \rightarrow K$, seen as an object of $\mathcal{K}^{2}$. The 2-functor $\mathrm{Cmd}(\mathrm{alg})$ takes the comonad $((K, t),(c, \lambda))$ in $\operatorname{Mnd}(\mathcal{K})$ to a comonad $\left(K^{t}=\operatorname{alg}(K, t), \bar{c}:=\operatorname{alg}(c, \lambda)\right)$ in $\mathcal{K}$, which is a lifting of the comonad $(K, c)$ to the Eilenberg-Moore object $K^{t}$ of $(K, t)$.

Similarly, if $\mathcal{K}$ admits Eilenberg-Moore constructions for comonads; that is, the fully faithful inclusion 2-functor $I_{*}: \mathcal{K} \rightarrow \mathrm{Cmd}(\mathcal{K})$ has a right 2-adjoint coalg, then there is a fully faithful 2-functor $\mathrm{Cmd}(\mathcal{K}) \rightarrow \mathcal{K}^{2}$ sending a comonad $(K, c)$ to the forgetful map $u^{c}: K^{c} \rightarrow K$. Once again, the 2-functor Mnd(coalg) takes the monad $((K, c),(t, \lambda))$ in $\operatorname{Cmd}(\mathcal{K})$ to a $\operatorname{monad}\left(K^{c}=\operatorname{coalg}(K, c), \bar{t}=\operatorname{coalg}(t, \lambda)\right)$ which is a lifting of the monad $(K, t)$ to the Eilenberg-Moore object $K^{c}$ of $(K, c)$.

If $\mathcal{K}$ admits Eilenberg-Moore constructions for both monads and comonads then there is a commutative diagram of fully faithful 2-functors

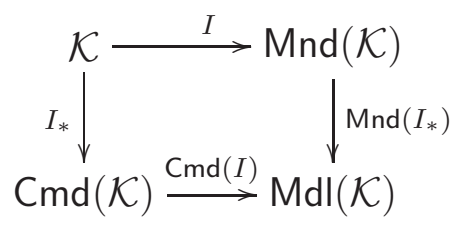

and since both alg $\circ \mathrm{Mnd}(\mathrm{coalg})$ and coalg $\circ \mathrm{Cmd}(\mathrm{alg})$ are right adjoint to the common diagonal in this last displayed diagram, they are naturally isomorphic, sending an object $(K, t, c, \lambda)$ of $\operatorname{Mdl}(\mathcal{K})$ to $\left(K^{c}\right)^{\bar{t}}$, respectively to $\left(K^{t}\right)^{\bar{c}}$. We shall sometimes write $K^{(t, c)}$ for this common value, although really $\lambda$ should be included in the notation. There are now fully faithful 2-functors

$$
\operatorname{Mdl}(\mathcal{K})=\operatorname{Mnd}(\operatorname{Cmd}(\mathcal{K})) \longrightarrow \operatorname{Mnd}\left(\mathcal{K}^{2}\right) \longrightarrow\left(\mathcal{K}^{2}\right)^{2}=\mathcal{K}^{2 \times 2}
$$

sending an object $(K, t, c, \lambda)$ of $\operatorname{Mdl}(\mathcal{K})$ to the commutative square of forgetful maps:

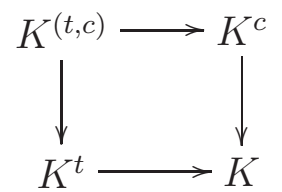

If a mixed distributive law in the 2-category Cat of Categories; Functors; Natural Transformations is induced by an entwining structure in Bim, then the associated category $K^{(t, c)}$ is known as the category of 'entwined modules' [6].

The situation of distributive laws between two monads is not completely analogous to the mixed case above. If $\mathcal{K}$ admits Eilenberg-Moore constructions for monads, then there is still a fully faithful 2-functor from $\operatorname{Mnd}(\operatorname{Mnd}(\mathcal{K}))$ - considered as the 2 -category of distributive laws between two monads - to $\mathcal{K}^{\mathbf{2} \times \mathbf{2}}$. The main difference in this "non-mixed" case is that while one of the monads lifts to the Eilenberg-Moore object of the other, the other extends to the Kleisli object of the first.

In order to treat algebra extensions by weak bialgebras [4, entwining structures were generalized in 8f to 'weak entwining structures', which are better called 'weak mixed distributive laws' if working in general 2-categories. A weak mixed distributive law in a 2-category $\mathcal{K}$ also consists of a monad $(K, t)$ and a comonad $(K, c)$, together with a 2-cell $t c \rightarrow c t$, but the compatibility axioms with the unit of the monad and the counit of the comonad are weakened. The corresponding notion of weak distributive law between two monads is discussed in [14]. The aim of this paper is to extend to 
weak (mixed) distributive laws the standard results for ordinary (mixed) distributive laws sketched above.

We are not aware of any characterization of a (mixed) weak distributive law as a monad (or as a comonad) in some 2-category. Instead, in this note we observe that a mixed weak distributive law in an arbitrary 2 -category $\mathcal{K}$ can be described as a compatible pair consisting of a comonad in a 2-category $\operatorname{Mnd}^{\iota}(\mathcal{K})$, extending $\operatorname{Mnd}(\mathcal{K})$, and a monad in $\operatorname{Cmd}^{\pi}(\mathcal{K}):=\operatorname{Mnd}^{\iota}\left(\mathcal{K}_{*}\right)_{*}$, cf. [3]. This observation is used in Section 1 ] to define a 2-category $\mathrm{Wdl}(\mathcal{K})$, whose 0 -cells are weak mixed distributive laws in $\mathcal{K}$ and whose 1-cells and 2-cells are also compatible pairs of 1-cells and 2-cells, respectively, in $\operatorname{Mnd}\left(\mathrm{Cmd}^{\pi}(\mathcal{K})\right)$ and $\mathrm{Cmd}\left(\mathrm{Mnd}^{\iota}(\mathcal{K})\right)$. By construction, the 2-category $\operatorname{Wdl}(\mathcal{K})$ comes equipped with 2-functors $\operatorname{Wdl}(\mathcal{K}) \rightarrow \operatorname{Cmd}\left(\operatorname{Mnd}^{\iota}(\mathcal{K})\right)$ and $\operatorname{Wdl}(\mathcal{K}) \rightarrow \operatorname{Mnd}\left(\mathrm{Cmd}^{\pi}(\mathcal{K})\right)$, and indeed $\operatorname{Wdl}(\mathcal{K})$ can be seen as a sort of "intersection" of $\operatorname{Mnd}\left(\mathrm{Cmd}^{\pi}(\mathcal{K})\right)$ and $\mathrm{Cmd}\left(\operatorname{Mnd}^{\iota}(\mathcal{K})\right)$.

Although the 2-categories $\mathrm{Mnd}^{\iota}(\mathcal{K})$ and $\mathrm{Cmd}^{\pi}(\mathcal{K})$ do not embed in $\mathcal{K}^{2}$, they embed in a sort of "weak" version of $\mathcal{K}^{2}$, corresponding to the "weak liftings" studied in [3] and [11]. Perhaps surprisingly, the 2 -category $\operatorname{Wdl}(\mathcal{K})$ does still embed in $\mathcal{K}^{\mathbf{2} \times \mathbf{2}}$. We prove this, and characterize the image of the embedding, as well as describing how this relates to the weak liftings described above.

If a weak mixed distributive law in Cat is induced by a weak entwining structure between a $k$-algebra $t$ and a $k$-coalgebra $c$, then the four objects occurring in its image in $\mathrm{Cat}^{\mathbf{2} \times \mathbf{2}}$ are the category of $k$-modules, the category of $t$-modules, the category of $c$-comodules and the category of so-called weak entwined modules [8], [5]. Important examples of weak entwining structures are associated with Doi-Koppinen data over weak bialgebras [2]. The corresponding weak entwined modules include various Hopf type modules over weak bialgebras - such as (relative) Hopf modules and YetterDrinfel'd modules - so in particular graded modules over groupoid graded algebras (cf.[9]). More exotic weak distributive laws, behind which there are no Doi-Koppinen data, were constructed in [7].

By a formal dualization of the above results on weak mixed distributive laws, one can also define a 2-category whose 0-cells are weak distributive laws between two monads. If Eilenberg-Moore constructions for monads exist and also idempotent 2cells split in $\mathcal{K}$, then we obtain a fully faithful 2 -functor from it to $\mathcal{K}^{\mathbf{2} \times \mathbf{2}}$.

Notation. We assume that the reader is familiar with the basic theory of 2categories. For a review of the required notions (such as 2-categories, 2-functors, 2adjunctions, monads, adjunctions and Eilenberg-Moore construction in a 2-category) we refer to the article [10]. In a 2-category $\mathcal{K}$, horizontal composition is denoted by juxtaposition and vertical composition is denoted by a dot. We say that in $\mathcal{K}$ idempotent 2-cells split provided that for any 2-cell $\Theta: V \rightarrow V$ such that $\Theta . \Theta=\Theta$, there exist a 1-cell $\widehat{V}$ and 2-cells $\pi: V \rightarrow \widehat{V}$ and $\iota: \widehat{V} \rightarrow V$, such that $\pi . \iota=\widehat{V}$ and $\iota . \pi=\Theta$.

Acknowledgement. GB would like to thank the organizers of the Conference in Hopf algebras and noncommutative algebra in the honor of Mia Cohen, in Sde-Boker, May 2010, for a generous invitation and an unforgettable first time in Israel. She also acknowledges financial support of the Hungarian Scientific Research Fund OTKA, grant no. F67910. All authors are grateful for partial support from the Australian Research Council, project DP0771252. 


\section{The 2-CATEgory OF MiXED WEAK Distributive LAWS}

A mixed weak distributive law in a 2-category $\mathcal{K}$ consists of a monad $(t, \mu, \eta)$ and a comonad $(c, \delta, \varepsilon)$ on an object $K$ of $\mathcal{K}$, along with a 2-cell $\lambda: t c \rightarrow c t$ making the following diagrams commute:

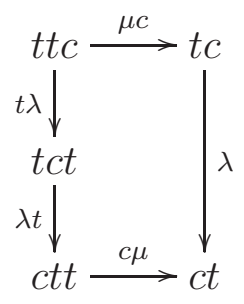

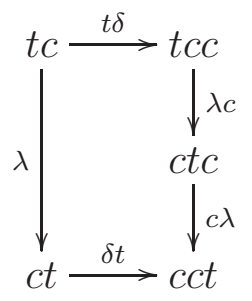
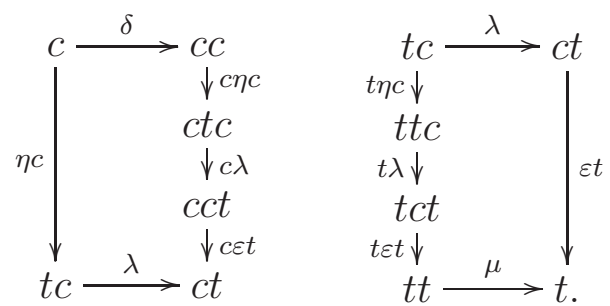

A 2-functor, or more generally a pseudofunctor, $F: \mathcal{K} \rightarrow \mathcal{L}$ sends weak distributive laws in $\mathcal{K}$ to weak distributive laws in $\mathcal{L}$. In particular, a representable 2 -functor $\mathcal{K}(X,-)$ sends a weak distributive law as above to a weak distributive law in Cat on the category $\mathcal{K}(X, K)$.

The main difference between mixed distributive laws and weak ones is that $(c, \lambda)$ is no longer a morphism of monads from $(K, t)$ to $(K, t)$, and so no longer induces a lifting $\bar{c}: K^{t} \rightarrow K^{t}$ when the Eilenberg-Moore object $K^{t}$ exists. Nonetheless, we shall see that $(c, \lambda)$ does induce a weak lifting $\bar{c}: K^{t} \rightarrow K^{t}$; equivalently, $(c, \lambda)$ is a weak morphism of monads $(K, t) \rightarrow(K, t)$ [3]. These weak morphisms of monads are the 1 -cells of a 2-category $\operatorname{Mnd}^{\iota}(\mathcal{K})$ whose objects are monads in $\mathcal{K}$.

Theorem 1.1 ([3], Corollary 1.4). For any 2-category $\mathcal{K}$, the following data constitute a 2-category, to be denoted by $\mathrm{Mnd}^{2}(\mathcal{K})$.

$\underline{0 \text {-cells }}$ are monads $(K, t)$ in $\mathcal{K}$.

$\underline{1 \text {-cells }}(K, t) \rightarrow\left(K^{\prime}, t^{\prime}\right)$ are pairs, consisting of a 1-cell $x: K \rightarrow K^{\prime}$ and a 2-cell $\xi: t^{\prime} x \rightarrow x t$ in $\mathcal{K}$ such that the first diagram in (1.2) commutes.

$\underline{\text { Q-cells }}(x, \xi) \rightarrow\left(x^{\prime}, \xi^{\prime}\right)$ are 2 -cells $\omega: x \rightarrow x^{\prime}$ in $\mathcal{K}$, rendering commutative the second diagram in (1.2).

Horizontal and vertical compositions are the same as in $\mathcal{K}$.

The 2-category $\mathrm{Mnd}^{\iota}(\mathcal{K})$ contains $\operatorname{Mnd}(\mathcal{K})$ as a vertically full 2-subcategory.
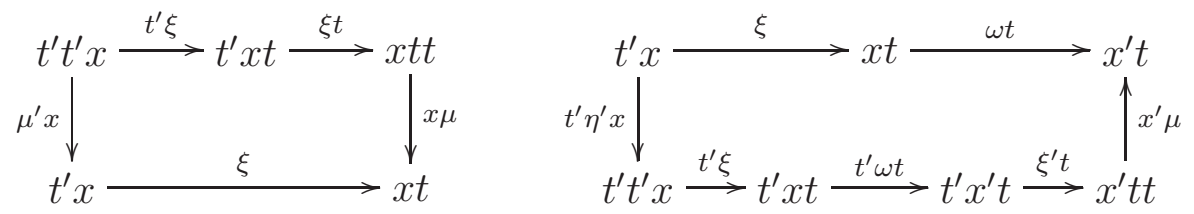

In [3, Corollary 1.4] another 2-category $\operatorname{Mnd}^{\pi}(\mathcal{K})$ was introduced, with the same 0and 1-cells as in $\operatorname{Mnd}^{\iota}(\mathcal{K})$ but different 2-cells. $\operatorname{Mnd}^{\pi}(\mathcal{K})$ also contains $\operatorname{Mnd}(\mathcal{K})$ as a vertically full 2-subcategory.

Similarly, for a weak mixed distributive law $(K, t, c, \lambda)$, we have only a weak morphism of comonads $(t, \lambda):(K, c) \rightarrow(K, c)$, and only a weak lifting of $t$ to $K^{c}$. There is a 2-category $\mathrm{Cmd}^{\pi}(\mathcal{K})=\operatorname{Mnd}^{\iota}\left(\mathcal{K}_{*}\right)_{*}$ of comonads in $\mathcal{K}$ and weak morphisms of comonads; once again, it contains $\mathrm{Cmd}(\mathcal{K})$ as a vertically full sub-2-category.

Our aim is to construct a 2-category of weak mixed distributive laws in any 2category $\mathcal{K}$; that is, a 2 -category $\operatorname{Wdl}(\mathcal{K})$ whose objects are weak mixed distributive laws. Our starting point is the following lemma. 
Lemma 1.2. For a monad $(K, t)$, a comonad $(K, c)$ and a 2-cell $\lambda: t c \rightarrow$ ct in any 2-category $\mathcal{K}$, the following statements are equivalent.

(i) $(K, t, c, \lambda)$ is a weak mixed distributive law;

(ii) $((K, t),(c, \lambda))$ is a comonad in $\operatorname{Mnd}^{\iota}(\mathcal{K})$ and $((K, c),(t, \lambda))$ is a monad in $\mathrm{Cmd}^{\pi}(\mathcal{K})$.

Proof. The first axiom in (1.1) expresses the requirement that $(c, \lambda):(K, t) \rightarrow(K, t)$ is a 1-cell in $\operatorname{Mnd}^{2}(\mathcal{K})$ and the second axiom in (1.1) means that $(t, \lambda):(K, c) \rightarrow(K, c)$ is a 1 -cell in $\mathrm{Cmd}^{\pi}(\mathcal{K})$. The third axiom in (1.1) means that $\eta: 1_{(K, c)} \rightarrow(t, \lambda)$ is a 2-cell in $\mathrm{Cmd}^{\pi}(\mathcal{K})$ and the last axiom in (1.1) means that $\varepsilon:(c, \lambda) \rightarrow 1_{(K, t)}$ is a 2-cell in $\operatorname{Mnd}^{\iota}(\mathcal{K})$. If these four conditions hold, then also $\mu:(t, \lambda)(t, \lambda) \rightarrow(t, \lambda)$ is a 2-cell in $\mathrm{Cmd}^{\pi}(\mathcal{K})$. That is, the following diagram commutes.

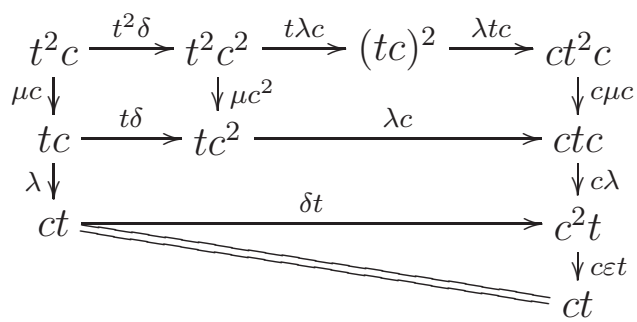

The top right region commutes by the first axiom, and the region below it commutes by the second axiom in (1.1). The top left square commutes by naturality and the triangle commutes by a counitality axiom of a comonad. Symmetrically, (1.1) implies that $\delta:(c, \lambda) \rightarrow(c, \lambda)(c, \lambda)$ is a 2-cell in $\operatorname{Mnd}^{\iota}(\mathcal{K})$.

The situation in Lemma 1.2 (ii) is distinguished among the other possibilities in the following sense.

Lemma 1.3. For a monad $(K, t)$, a comonad $(K, c)$, and a 2-cell $\lambda: t c \rightarrow c t$ in any 2-category $\mathcal{K}$, the following assertions are equivalent.

(i) $(K, t, c, \lambda)$ is mixed distributive law;

(ii) $((K, t),(c, \lambda))$ is a comonad in $\operatorname{Mnd}(\mathcal{K})$;

(iii) $((K, c),(t, \lambda))$ is a monad in $\operatorname{Cmd}(\mathcal{K})$;

(iv) $((K, c),(t, \lambda))$ is a monad in $\mathrm{Cmd}^{\iota}(\mathcal{K})$ and $((K, t),(c, \lambda))$ is a comonad in $\mathrm{Mnd}^{\pi}(\mathcal{K})$;

(v) $((K, c),(t, \lambda))$ is a monad in $\mathrm{Cmd}^{\pi}(\mathcal{K})$ and $((K, t),(c, \lambda))$ is a comonad in $\operatorname{Mnd}^{\pi}(\mathcal{K})$

(vi) $((K, c),(t, \lambda))$ is a monad in $\mathrm{Cmd}^{\iota}(\mathcal{K})$ and $((K, t),(c, \lambda))$ is a comonad in $\operatorname{Mnd}^{\iota}(\mathcal{K})$.

Proof. Equivalence of (i), (ii) and (iii) is well-known, see e.g. [12, Proposition 6.3 and Corollary 6.6]. Assertions (ii) and (iii) trivially imply any of (iv), (v) and (vi). The counit $\varepsilon$ of $(K, c)$ is a 2-cell $(c, \lambda) \rightarrow 1_{(K, t)}$ in $\operatorname{Mnd}^{\pi}(\mathcal{K})$ if and only if $\varepsilon t . \lambda=t \varepsilon$ and the unit $\eta$ of $(K, t)$ is a 2-cell $1_{(K, c)} \rightarrow(t, \lambda)$ in $\mathrm{Cmd}^{\iota}(\mathcal{K})$ if and only if it $\lambda . \eta c=c \eta$. Hence (iv) $\Rightarrow(\mathrm{i})$. If (v) holds then $\lambda$ obeys the third axiom in (1.1) and $\varepsilon t . \lambda=t \varepsilon$, hence by counitality of $\delta$ also $\lambda . \eta c=c \eta$. This proves $(\mathrm{v}) \Rightarrow(\mathrm{i})$ and $(\mathrm{vi}) \Rightarrow(\mathrm{i})$ follows symmetrically.

The next two lemmas are preparatory to our definition of 1-cells and 2-cells in the 2-category $\operatorname{Wdl}(\mathcal{K})$. 
Lemma 1.4. For weak mixed distributive laws $(K, t, c, \lambda)$ and $\left(K^{\prime}, t^{\prime}, c^{\prime}, \lambda^{\prime}\right)$ in a 2category $\mathcal{K}$, consider a 1 -cell $(x, \xi):(K, t) \rightarrow\left(K^{\prime}, t^{\prime}\right)$ in $\operatorname{Mnd}(\mathcal{K})$ and a 1 -cell $(x, \zeta)$ : $(K, c) \rightarrow\left(K^{\prime}, c^{\prime}\right)$ in $\operatorname{Cmd}(\mathcal{K})$. The following are equivalent.

(i) $\xi:\left(t^{\prime}, \lambda^{\prime}\right)(x, \zeta) \rightarrow(x, \zeta)(t, \lambda)$ is a 2-cell in $\mathrm{Cmd}^{\pi}(\mathcal{K})$; that is, the following diagram commutes;

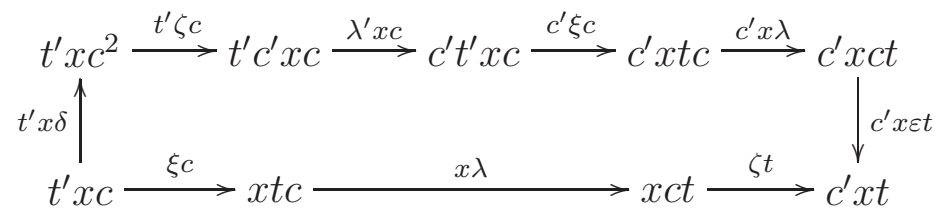

(ii) $\zeta:(x, \xi)(c, \lambda) \rightarrow\left(c^{\prime}, \lambda^{\prime}\right)(x, \xi)$ is a 2-cell in $\operatorname{Mnd}^{\prime}(\mathcal{K})$; that is, the following diagram commutes.

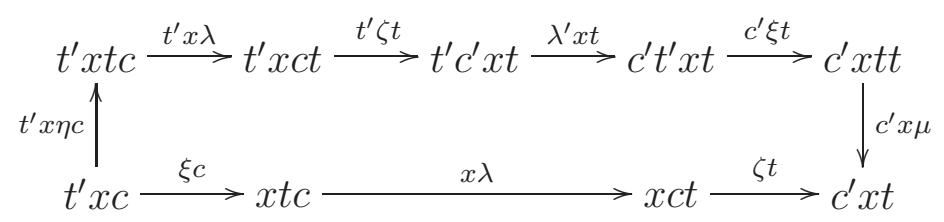

When these conditions hold, we say that $(x, \xi, \zeta)$ is a morphism of weak distributive laws from $(K, t, c, \lambda)$ to $\left(K^{\prime}, t^{\prime}, c^{\prime}, \lambda^{\prime}\right)$.

Proof. This follows by commutativity of

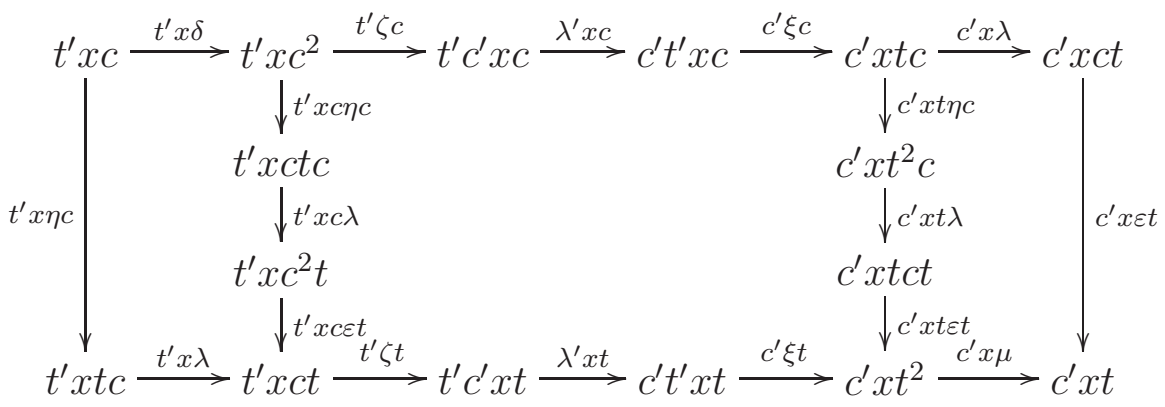

in which the large central region commutes by naturality, and the other two regions by the weak distributive law axioms.

Lemma 1.5. For morphisms $(x, \xi, \zeta),\left(x^{\prime}, \xi^{\prime}, \zeta^{\prime}\right):(K, t, c, \lambda) \rightarrow\left(K^{\prime}, t^{\prime}, c^{\prime}, \lambda^{\prime}\right)$ of weak distributive laws in a 2-category $\mathcal{K}$, and a 2-cell $\omega: x \rightarrow x^{\prime}$, the following conditions are equivalent.

(i) $\omega:(x, \xi) \rightarrow\left(x^{\prime}, \xi^{\prime}\right)$ is a 2-cell in $\operatorname{Mnd}(\mathcal{K})$ and $\omega:(x, \zeta) \rightarrow\left(x, \zeta^{\prime}\right)$ is a 2-cell in $\mathrm{Cmd}(\mathcal{K})$

(ii) $\omega:((x, \xi), \zeta) \rightarrow\left(\left(x^{\prime}, \xi^{\prime}\right), \zeta^{\prime}\right)$ is a 2 -cell in $\mathrm{Cmd}\left(\mathrm{Mnd}^{\iota}(\mathcal{K})\right)$ and $\omega:((x, \zeta), \xi) \rightarrow$ $\left(\left(x^{\prime}, \zeta^{\prime}\right), \xi^{\prime}\right)$ is a 2-cell in $\mathrm{Mnd}\left(\mathrm{Cmd}^{\pi}(\mathcal{K})\right)$.

We then say that $\omega$ is a 2-cell $(x, \xi, \zeta) \rightarrow\left(x^{\prime}, \xi^{\prime}, \zeta^{\prime}\right)$ of weak distributive laws.

Proof. In each case the conditions assert the commutativity of the squares
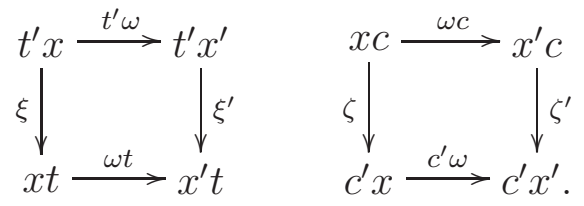
We now deduce:

Theorem 1.6. For any 2-category $\mathcal{K}$, the weak distributive laws in $\mathcal{K}$ along with the morphisms and 2-cells defined above give a 2-category $\mathrm{Wdl}(\mathcal{K})$ with composition performed as in $\operatorname{Mnd}(\mathcal{K})$ and $\mathrm{Cmd}(\mathcal{K})$.

\section{A Fully faithful EMBEDding FOR WEAK MiXED DistribUtive LAWS}

For any 2-category $\mathcal{K}$, there is a fully faithful 2-functor $Y: \mathcal{K} \rightarrow \operatorname{Wdl}(\mathcal{K})$ which equips any object $X$ with the identity monad, the identity comonad, and the identity distributive law between them; we write $Y X=(X, 1,1,1)$.

If $(K, t, c, \lambda)$ is any weak distributive law, a morphism in $\mathrm{Wdl}(\mathcal{K})$ from $Y X$ to $(K, t, c, \lambda)$ consists of a morphism $a: X \rightarrow K$ in $\mathcal{K}$, equipped with an action $\alpha: t a \rightarrow a$ of the monad $t$ and a coaction $\gamma: a \rightarrow c a$ of the comonad $c$, satisfying the compatibility condition asserting that the diagram

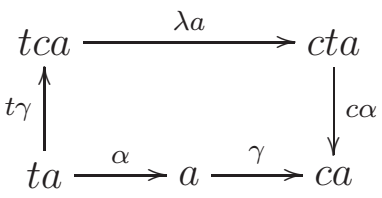

commutes. We then say that $(a, \alpha, \gamma)$ is a mixed $(K, t, c, \lambda)$-algebra with domain $X$. A morphism of mixed $(K, t, c, \lambda)$-algebras is a 2 -cell in $\mathcal{K}$ compatible with the action and coaction. Thus the category of mixed algebras with domain $X$ is the hom-category

$$
\operatorname{Wdl}(\mathcal{K})(Y X,(K, t, c, \lambda)) \text {. }
$$

In particular, we may take $\mathcal{K}=$ Cat and $X=1$, the terminal category. Then $a$ is just an object of $\mathcal{K}$, and the action and coaction amount to a $t$-algebra structure and a $c$-coalgebra structure in the usual sense, while the compatibility condition has exactly the form of the diagram displayed above. We then write $K^{(t, c)}$ for the category of mixed $(K, t, c, \lambda)$-algebras.

In fact we can also recover the general notion of mixed algebra from this particular one: for a weak distributive law $(K, t, c, \lambda)$ in a 2-category $\mathcal{K}$, applying the representable 2-functor $\mathcal{K}(X,-): \mathcal{K} \rightarrow$ Cat gives a weak distributive law

$$
(\mathcal{K}(X, K), \mathcal{K}(X, t), \mathcal{K}(X, c), \mathcal{K}(X, \lambda))
$$

in Cat whose category of mixed algebras is just the category of mixed $(K, t, c, \lambda)$ algebras with domain $X$. This passage from $X$ to the category of mixed $(K, t, c, \lambda)$ algebras with domain $X$ defines a 2-functor $\mathcal{K}^{\mathrm{op}} \rightarrow$ Cat, and if this 2-functor is representable, say as

$$
\mathcal{K}(X, K)^{(\mathcal{K}(X, t), \mathcal{K}(X, c))} \cong \mathcal{K}\left(X, K^{(t, c)}\right)
$$

we call the representing object $K^{(t, c)}$ the mixed Eilenberg-Moore object. (Clearly, if $\mathcal{K}=$ Cat, we re-cover the above category $K^{(t, c)}$ of mixed $(K, t, c, \lambda)$-algebras with domain 1.)

Theorem 2.1. $\mathcal{K}$ has mixed Eilenberg-Moore objects if and only if $Y: \mathcal{K} \rightarrow \operatorname{Wdl}(\mathcal{K})$ has a right 2-adjoint. 
Proof. This follows from the isomorphism

$$
\operatorname{Wdl}(\mathcal{K})(Y X,(K, t, c, \lambda)) \cong \mathcal{K}(X, K)^{(\mathcal{K}(X, t), \mathcal{K}(X, c))}
$$

since the mixed Eilenberg-Moore object is defined as a representing object for the right hand side, while the right adjoint is defined as a representing object for the left hand side.

Theorem 2.2. Suppose that $\mathcal{K}$ has Eilenberg-Moore objects for monads and comonads, and that idempotent 2-cells split. Then $\mathcal{K}$ has mixed Eilenberg-Moore objects.

Proof. Form the Eilenberg-Moore object $K^{c}$. Since $(t, \lambda)$ is a monad in $\mathrm{Cmd}^{\pi}(\mathcal{K})$, it has a weak lifting to a monad $\bar{t}$ on $K^{c}$ cf. [3, Proposition 5.7]. The EilenbergMoore object $\left(K^{c}\right)^{\bar{t}}$ is the desired mixed Eilenberg-Moore object. Symmetrically, the comonad $(c, \lambda)$ has a weak lifting to a monad $\bar{c}$ on $K^{t}$, and $\left(K^{t}\right)^{\bar{c}}$ also gives the mixed Eilenberg-Moore object.

Theorem 2.3. Suppose that $\mathcal{K}$ has Eilenberg-Moore objects for monads and comonads and that idempotent 2-cells split. Then $\operatorname{WdI}(\mathcal{K})$ has a fully faithful embedding into $\mathcal{K}^{2 \times 2}$.

Proof. The embedding will send an object $(K, t, c, \lambda)$ to the square

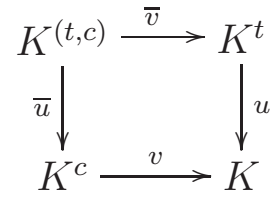

of Eilenberg-Moore objects and forgetful morphisms. In order to conclude that it extends to the stated embedding, we need to establish isomorphisms between the respective hom categories of $\operatorname{Wdl}(\mathcal{K})$ and $\mathcal{K}^{\mathbf{2} \times \mathbf{2}}$.

A 1-cell in $\operatorname{Wdl}(\mathcal{K})$ consists of a compatible pair of a monad morphism and a comonad morphism. If $\left(K^{\prime}, t^{\prime}, c^{\prime}, \lambda^{\prime}\right)$ is another object of $\operatorname{Wdl}(\mathcal{K})$, then consider a morphism $x: K \rightarrow K^{\prime}$. To give a monad morphism $(x, \xi):(K, t) \rightarrow\left(K^{\prime}, t^{\prime}\right)$ is equivalently to give a lifting $x^{\xi}: K^{t} \rightarrow K^{\prime t^{\prime}}$ of $x$; this in turn is equivalent to the fact that for any $t$-algebra with domain $X$, consisting of a morphism $a: X \rightarrow K$ with an action $\alpha: t a \rightarrow a$, the composite

$$
t^{\prime} x a \stackrel{\xi a}{\longrightarrow} x t a \stackrel{x \alpha}{\longrightarrow} x a
$$

makes $x a$ into a $t^{\prime}$-algebra with domain $X$. Similarly, to give a comonad morphism $(x, \zeta):(K, c) \rightarrow\left(K^{\prime}, c^{\prime}\right)$ is equivalently to give a lifting $x^{\zeta}: K^{c} \rightarrow K^{\prime c^{\prime}}$ of $x$; this is equivalent to the fact that for any $c$-coalgebra $(a, \gamma)$ with domain $X$, the composite

$$
x a \stackrel{x \gamma}{\longrightarrow} x c a \stackrel{\zeta a}{\longrightarrow} c^{\prime} x a
$$

makes $x a$ into a $c^{\prime}$-coalgebra with domain $X$.

We should check that $(x, \xi, \zeta)$ is a morphism in $\operatorname{Wdl}(\mathcal{K})$ if and only if $x^{\xi}$ and $x^{\zeta}$ have a common lifting $x^{(\xi, \zeta)}: K^{(t, c)} \rightarrow K^{\prime\left(t^{\prime}, c^{\prime}\right)}$ (giving rise to a 1 -cell in $\mathcal{K}^{2 \times 2}$ ); in other words, if and only for any mixed $(K, t, c, \lambda)$-algebra $(a, \alpha, \gamma)$ with domain $X$, the induced $t^{\prime}$-algebra and $c^{\prime}$-coalgebra structures on $x a$ together give a $\left(K^{\prime}, t^{\prime}, c^{\prime}, \lambda^{\prime}\right)$ algebra. But we can regard $(a, \alpha, \gamma)$ as a morphism in $\operatorname{Wdl}(\mathcal{K})$ from $Y X$ to $(K, t, c, \lambda)$, 
and now composing with $(x, \xi, \zeta)$ gives a morphism $Y X \rightarrow\left(K^{\prime}, t^{\prime}, c^{\prime}, \lambda^{\prime}\right)$ which gives the desired $\left(K^{\prime}, t^{\prime}, c^{\prime}, \lambda^{\prime}\right)$-algebra.

Conversely, suppose that $x^{\xi}$ and $x^{\zeta}$ have a common lifting $x^{(\xi, \zeta)}$. The composite

$$
t c \stackrel{t \delta}{\longrightarrow} t c^{2} \stackrel{\lambda c}{\longrightarrow} c t c \stackrel{\varepsilon t c}{\longrightarrow} t c
$$

is idempotent, and splits to give a morphism $a: K \rightarrow K$ equipped with an epimorphism $\pi: t c \rightarrow a$ and monomorphism $\sigma: a \rightarrow t c$. Then $a$ has a mixed $(K, t, c, \lambda)$ algebra structure $(a, \alpha, \gamma)$ coming from

$$
t a \stackrel{t \sigma}{\longrightarrow} t^{2} c \stackrel{\mu c}{\longrightarrow} t c \stackrel{\pi}{\longrightarrow} a \text { and } a \stackrel{\sigma}{\longrightarrow} t c \stackrel{t \delta}{\longrightarrow} t c^{2} \stackrel{\lambda c}{\longrightarrow} c t c \stackrel{c \pi}{\longrightarrow} c a .
$$

Applying the lifting $x^{(\xi, \zeta)}$ gives a mixed $\left(K^{\prime}, t^{\prime}, c^{\prime}, \lambda^{\prime}\right)$-algebra structure on $x a$ via the maps

$$
t^{\prime} x a \stackrel{\xi a}{\longrightarrow} x t a \stackrel{x \alpha}{\longrightarrow} x a \text { and } x a \stackrel{x \gamma}{\longrightarrow} x c a \stackrel{\zeta a}{\longrightarrow} c^{\prime} x a .
$$

Write $\eta_{1}: c \rightarrow a$ and $\varepsilon_{1}: a \rightarrow t$ for the composites

$$
c \stackrel{\eta c}{\longrightarrow} t c \stackrel{\pi}{\longrightarrow} a \quad \text { and } \quad a \stackrel{\sigma}{\longrightarrow} t c \stackrel{t \varepsilon}{\longrightarrow} t .
$$

In view of (1.3) $),(x, \xi, \zeta)$ will be a morphism in $\mathrm{Wdl}(\mathcal{K})$ provided that the exterior of

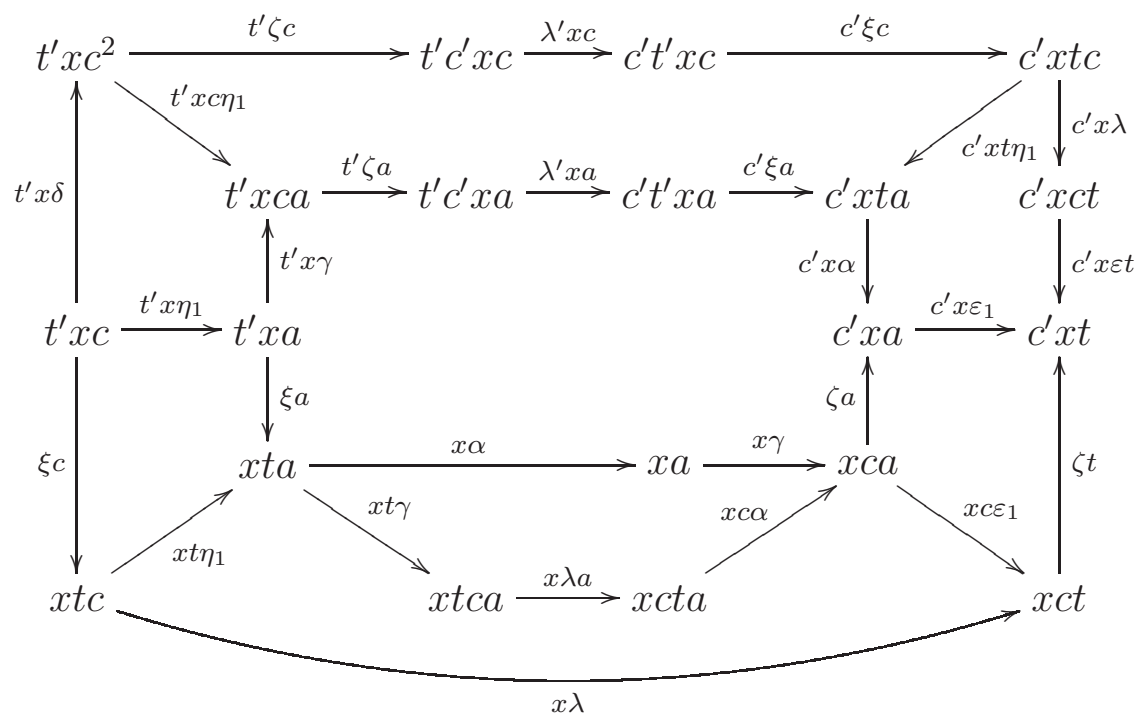

commutes. The central region commutes since $x a$ is a mixed algebra, the region just below it because $a$ is a mixed algebra, the top region and the lower corners by naturality. Thus we just need to show that the lower region and the two upper corners commute. These will follow from commutativity of the following three diagrams:

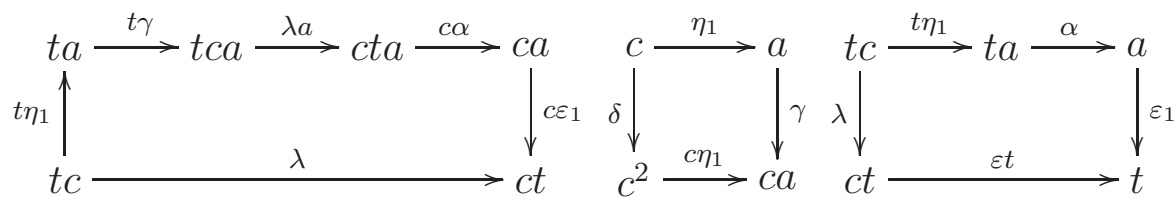

which involve a single weak distributive law ( $x$ does not appear). We prove them as a separate lemma below.

We now turn to fully faithfulness on 2-cells. Let $(x, \xi, \zeta)$ and $\left(x^{\prime}, \xi^{\prime}, \zeta^{\prime}\right)$ be 1-cells from $(K, t, c, \lambda)$ to $\left(K^{\prime}, t^{\prime}, c^{\prime}, \lambda^{\prime}\right)$, with induced liftings $x^{\xi}, x^{\prime \xi^{\prime}}$, and so on. A 2-cell 
$\omega: x \rightarrow x^{\prime}$ in $\mathcal{K}$ lifts to a 2-cell $\omega^{\xi}: x^{\xi} \rightarrow x^{\prime \xi^{\prime}}$ if and only if $\omega$ is a monad 2-cell $(x, \xi) \rightarrow\left(x^{\prime}, \xi^{\prime}\right)$; similarly it lifts to a 2-cell $\omega^{\zeta}: x^{\zeta} \rightarrow x^{\prime \zeta^{\prime}}$ if and only if it is a comonad 2 -cell $(x, \zeta) \rightarrow\left(x^{\prime}, \zeta^{\prime}\right)$. Suppose both of these conditions hold. There is no further condition for $\omega$ to be a 2-cell $(x, \xi, \zeta) \rightarrow\left(x^{\prime}, \xi^{\prime}, \zeta^{\prime}\right)$; thus it remains to show that $\omega^{\xi}$ and $\omega^{\zeta}$ have a common lifting $\omega^{(\xi, \zeta)}: x^{(\xi, \zeta)} \rightarrow x^{\prime\left(\xi^{\prime}, \zeta^{\prime}\right)}$ (giving rise to a 2 -cell in $\mathcal{K}^{2 \times 2}$ ). For this, it will suffice to show that for any mixed $(K, t, c, \lambda)$-algebra $(a, \alpha, \gamma)$, if we form the induced mixed $\left(K^{\prime}, t^{\prime}, c^{\prime}, \lambda^{\prime}\right)$-algebra structures on $x a$ and $x^{\prime} a$, then $\omega a: x a \rightarrow x^{\prime} a$ will be a morphism of mixed $\left(K^{\prime}, t^{\prime}, c^{\prime}, \lambda^{\prime}\right)$-algebras. This follows by regarding $(a, \alpha, \gamma)$ as a morphism $Y X \rightarrow(K, t, c, \lambda)$ in $\operatorname{Wdl}(\mathcal{K})$, and then composing this with the 2-cell $\omega:(x, \xi, \zeta) \rightarrow\left(x^{\prime}, \xi^{\prime}, \zeta^{\prime}\right)$. This gives a 2-cell $(x, \xi, \zeta)(a, \alpha, \gamma) \rightarrow\left(x^{\prime}, \xi^{\prime}, \zeta^{\prime}\right)(a, \alpha, \gamma)$ with domain $Y X$ and codomain $\left(K^{\prime}, t^{\prime}, c^{\prime}, \lambda^{\prime}\right)$, and this is the desired morphism of $\left(K^{\prime}, t^{\prime}, c^{\prime}, \lambda^{\prime}\right)$-algebras.

Lemma 2.4. For any weak mixed distributive law $(K, t, c, \lambda)$ in a 2-category $\mathcal{K}$ with split idempotent 2-cells, the diagrams in (2.2) commute.

Proof. In the diagrams
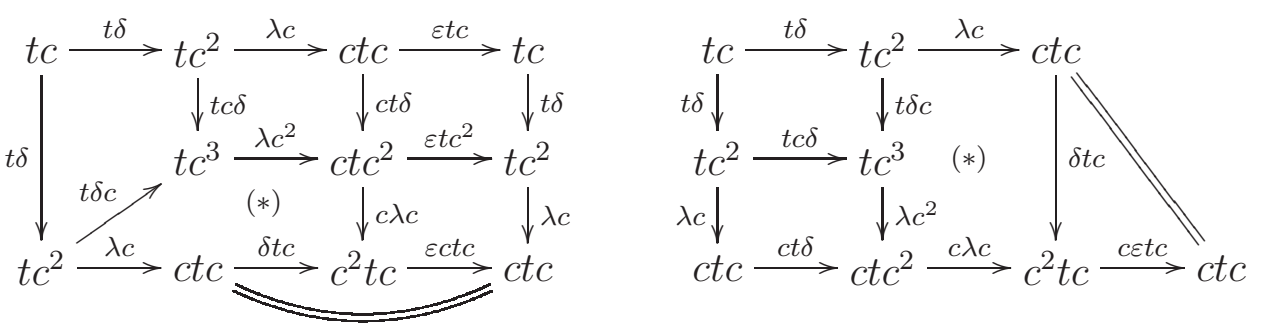

the marked regions commute by the second axiom of a weak mixed distributive law. The other regions commute by naturality, coassociativity and counitality of the comonad $c$. This proves that $\lambda c . t \delta . \sigma . \pi=\lambda c . t \delta=c \sigma . c \pi . \lambda c . t \delta$. Hence recalling from (2.1) the formula of $\gamma$, and composing the top-right path of the second diagram in (2.2) by the monomorphism $c \sigma$ at the end, we obtain the morphism

$$
\left(c \stackrel{\eta c}{\longrightarrow} t c \stackrel{t \delta}{\longrightarrow} t c^{2} \stackrel{\lambda c}{\longrightarrow} c t c\right)=\left(c \stackrel{\delta}{\longrightarrow} c^{2} \stackrel{\eta c^{2}}{\longrightarrow} t c^{2} \stackrel{\lambda c}{\longrightarrow} c t c\right) .
$$

In the following diagram the square on the right commutes by the third axiom of a weak mixed distributive law; the top left square commutes by coassociativity of $\delta$ and the bottom left square commutes by naturality.

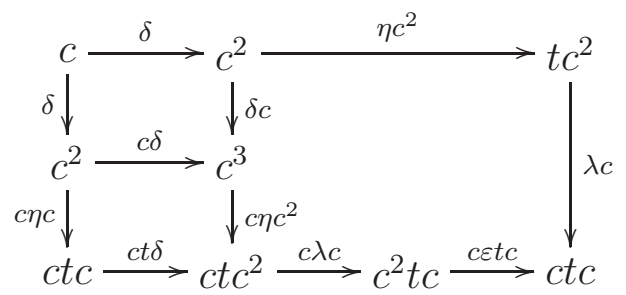

Hence composing by $c \sigma$ the left-bottom path of the second diagram in (2.2), we get the same morphism (2.3). This proves commutativity of the second diagram in (2.2). 
The diagrams
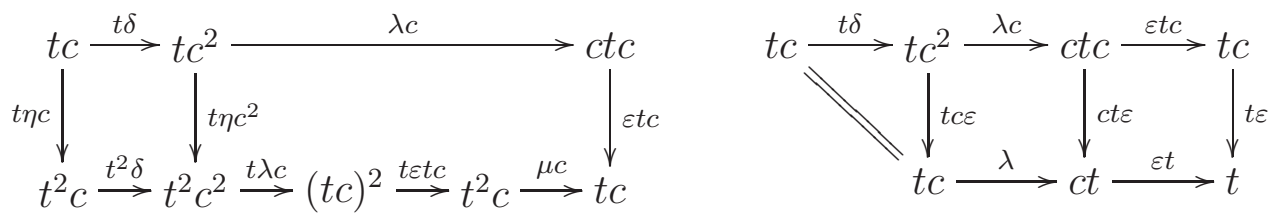

commute by the last axiom of a weak mixed distributive law; naturality and counitality of the comonad $c$. The equal paths around the first diagram describe an idempotent morphism. Thus recalling the expression of $\alpha$ from (2.1), the third diagram in (2.2) is seen to be commutative.

Finally, the first diagram in (2.2) is identical to the outer square in

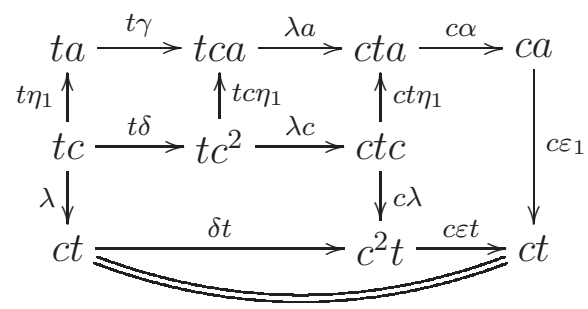

Its top left and right regions commute by the second and third diagrams in (2.2), respectively. The pentagon at the bottom left commutes by the second axiom of a weak mixed distributive law. The middle square at the top commutes by naturality and the bottom path is the identity morphism by the counitality of $c$.

\section{A CHARACTERIZATion OF THE IMAGE}

For the rest of the paper, we suppose that $\mathcal{K}$ is a 2-category with Eilenberg-Moore objects for monads and comonads, and splittings for idempotent 2-cells. We saw in the previous section that there is a fully faithful 2 -functor $\operatorname{Wdl}(\mathcal{K}) \rightarrow \mathcal{K}^{\mathbf{2} \times \mathbf{2}}$; in this section we characterize its image.

First recall that for an adjunction $f \dashv u$, as well as the bijection between 2-cells $f x \rightarrow y$ and $x \rightarrow u y$, there is also a bijection between 2-cells $m u \rightarrow n$ and $m \rightarrow n f$. Combining these two facts, we see that if also $\bar{f} \dashv \bar{u}$ is an adjunction, then there are bijections between 2-cells $f x \rightarrow y \bar{f}, 2$-cells $x \rightarrow u y \bar{f}$, and 2-cells $x \bar{u} \rightarrow u y$. A 2-cell $f x \rightarrow y \bar{f}$ and the corresponding 2-cell $x \bar{u} \rightarrow u y$ are said to be mates [10]. Explicitly, the mates of $\varphi: x \bar{u} \rightarrow u y$ and $\psi: f x \rightarrow y \bar{f}$ are the composites

$$
f x \stackrel{f x \bar{\eta}}{\longrightarrow} f x \bar{u} \bar{f} \stackrel{f \varphi \bar{f}}{\longrightarrow} f u y \bar{f} \stackrel{\varepsilon y \bar{f}}{\longrightarrow} y \bar{f} \quad x \bar{u} \stackrel{\eta x \bar{u}}{\longrightarrow} u f x \bar{u} \stackrel{u \psi \bar{u}}{\longrightarrow} u y \bar{f} \bar{u} \stackrel{u y \bar{\varepsilon}}{\longrightarrow} u y
$$

where as usual $\eta$ and $\varepsilon$ (possibly with a bar) denote the unit and counit of an adjunction.

Consider a commutative square

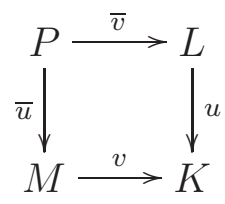

in $\mathcal{K}$, and suppose that we have adjunctions $f \dashv u, \bar{f} \dashv \bar{u}, v \dashv g$, and $\bar{v} \dashv \bar{g}$. 
The identity 2-cell $v \bar{u}=u \bar{v}$ has mates $\pi: f v \rightarrow \bar{v} \bar{f}$ and $\sigma: \bar{u} \bar{g} \rightarrow g u$ given by the following composites.

$$
f v \stackrel{f v \bar{\eta}}{\longrightarrow} f v \bar{u} \bar{f}=f u \bar{v} \bar{f} \stackrel{\varepsilon \bar{v} \bar{f}}{\longrightarrow} \bar{v} \bar{f} \quad \bar{u} \bar{g} \stackrel{\eta \bar{u} \bar{g}}{\longrightarrow} g v \bar{u} \bar{g}=g u \bar{v} \bar{g} \stackrel{g u \bar{\varepsilon}}{\longrightarrow} g u
$$

We shall use the following easy lemma:

Lemma 3.1. Let $\ell \dashv r$ and $\ell^{\prime} \dashv r^{\prime}$ be adjunctions, and $\alpha: \ell \rightarrow \ell^{\prime}$ a 2-cell with mate $\beta: r^{\prime} \rightarrow r$. Then $\check{\alpha}: \ell^{\prime} \rightarrow \ell$ satisfies $\alpha \check{\alpha}=1$ if and only if the mate $\hat{\beta}$ of $\check{\alpha}$ satisfies $\hat{\beta} \beta=1$.

Proposition 3.2. There is a bijection between 2 -cells $\check{\pi}: \bar{v} \bar{f} \rightarrow$ fv for which $\pi \check{\pi}=1$ and 2-cells $\hat{\sigma}: g u \rightarrow \bar{u} \bar{g}$ for which $\hat{\sigma} \sigma=1$.

Proof. The adjunctions $f \dashv u$ and $v \dashv g$ compose to give an adjunction $f v \dashv g u$. Similarly, the adjunctions $\bar{f} \dashv \bar{u}$ and $\bar{v} \dashv \bar{g}$ compose to give an adjunction $\bar{v} \dashv \bar{u} \bar{g}$. The result now follows immediately from the lemma.

Theorem 3.3. Let $\mathcal{K}$ be a 2-category with Eilenberg-Moore objects for monads and comonads and splittings for idempotent 2-cells. A commutative square

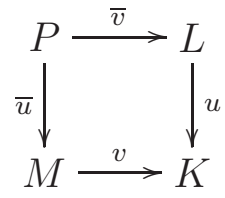

is in the image of the embedding $\mathrm{WdI}(\mathcal{K}) \rightarrow \mathcal{K}^{\mathbf{2} \times \mathbf{2}}$ if and only if $u$ and $\bar{u}$ are monadic, $v$ and $\bar{v}$ are comonadic, and the mate $\pi: f v \rightarrow \bar{v} \bar{f}$ (under the adjunctions $f \dashv u$ and $\bar{f} \dashv \bar{u}$ ) has a section $\check{\pi}$.

Proof. Since all these notions are preserved and jointly reflected by the representable functors $\mathcal{K}(X,-): \mathcal{K} \rightarrow$ Cat, it will suffice to prove the theorem in the case $\mathcal{K}=$ Cat. Suppose that $u$ and $\bar{u}$ are monadic, while $v$ and $\bar{v}$ are comonadic; this is certainly necessary for the square to be in the image. Denote the various adjoints as above, let $t$ be the monad induced by $f \dashv u$ and $c$ the comonad induced by $v \dashv g$.

Assume that the square is the image of some weak distributive law $(K, t, c, \lambda)$ and take an object $(a, \gamma)$ of $M=K^{c}$. Then $f v(a, \gamma)=(t a, \mu a)$, while $\bar{v} \bar{f}(a, \gamma)$ is obtained by splitting (that is, taking the image of) the idempotent

$$
t a \stackrel{t \gamma}{\longrightarrow} t c a \stackrel{\lambda a}{\longrightarrow} c t a \stackrel{\varepsilon t a}{\longrightarrow} t a .
$$

By the last of the diagrams defining a weak distributive law, this idempotent is in fact a morphism of $t$-algebras. The epimorphism appearing in the splitting is just the component at $(a, \gamma)$ of $\pi: f v \rightarrow \bar{v}$, and so we can take the other half of the splitting as $\check{\pi}$. This proves that any square in the image satisfies the stated conditions.

Suppose conversely that our square satisfies the stated condition. We have a monad $t$ and a comonad $c$ on $K$. We construct $\lambda: t c \rightarrow c t$ as the composite

$$
u f v g \stackrel{u \pi g}{\longrightarrow} u \bar{v} \bar{f} g=v \bar{u} \bar{f} g \stackrel{v \bar{u} \theta}{\longrightarrow} v \bar{u} \bar{g} f \stackrel{v \sigma f}{\longrightarrow} v g u f
$$


where $\theta: \bar{f} g \rightarrow \bar{g} f$ is the mate of $\check{\pi}$ under the adjunctions $v \dashv g$ and $\bar{v} \dashv \bar{g}$ (or alternatively, the mate of $\hat{\sigma}$ under $f \dashv u$ and $\bar{f} \dashv \bar{u}$ ). We must show that $\lambda$ is a weak distributive law, and that the category of mixed $(K, t, c, \lambda)$-algebras is $P$.

In the diagram

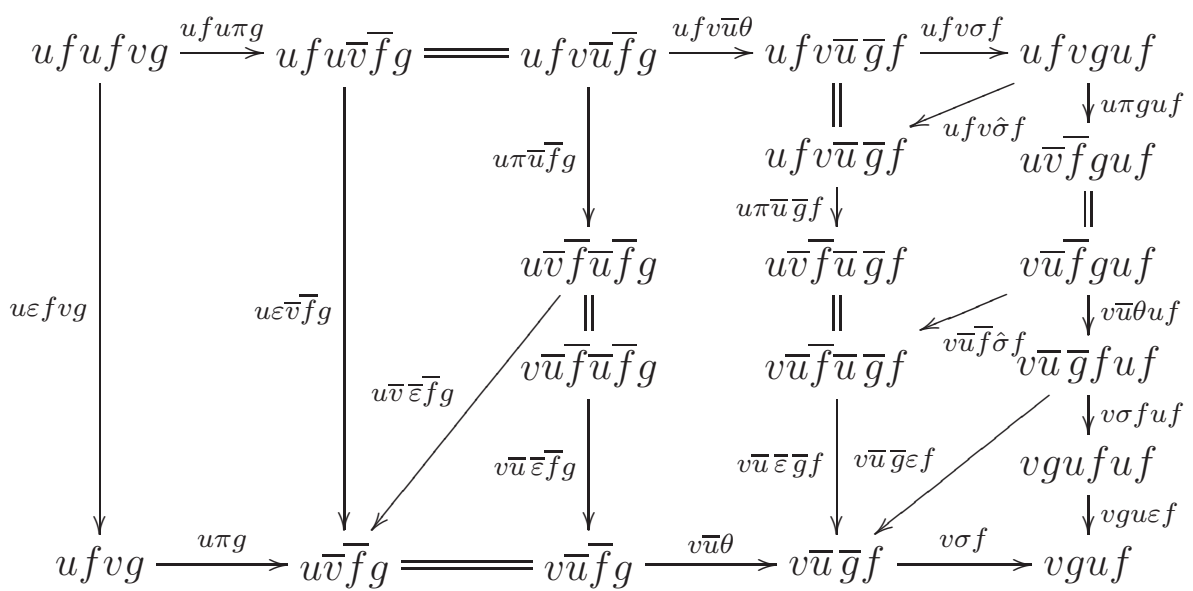

the triangle in the top right corner commutes since $\hat{\sigma} \sigma=1$, the remaining regions commute by naturality or by mateship relations, and so the exterior commutes, giving compatibility of $\lambda$ with $\mu$. Compatibility with $\delta$ is similar.

In the diagram

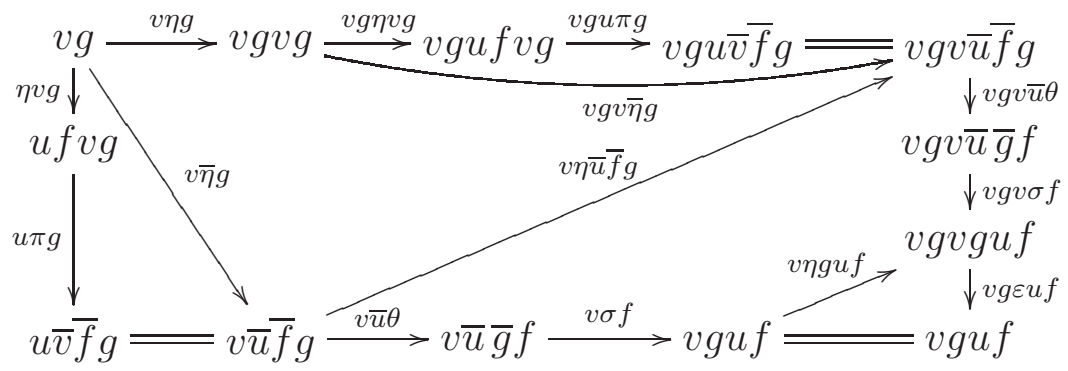

the triangle in the bottom right corner commutes by one of the triangle equations for the adjunction $v \dashv g$, the triangular region on the left and the narrow region at the top commute by mateship relations, the remaining regions commute by naturality, and so the exterior commutes, giving compatibility of $\lambda$ with $\eta$. Compatibility with $\varepsilon$ is similar.

Finally we should observe that the monad induced by the adjunction $\bar{f} \dashv \bar{u}$ is precisely the weakly lifted monad $\bar{t}$, and so $P$ is the category of $\bar{t}$-algebras, which in turn is the category of mixed $(K, t, c, \lambda)$-algebras.

Remark 3.4. Among the weak mixed distributive laws are the actual (non-weak) mixed distributive laws. They correspond to the case where $\pi: f v \rightarrow \bar{v} \bar{f}$ is not just a split epimorphism, but invertible. Since $f v \dashv g u$ and $\bar{v} \bar{f} \dashv \bar{u} \bar{g}$, this is of course equivalent to $\sigma: \bar{u} \bar{g} \rightarrow g u$ being invertible.

\section{Consequences}

In this final section we draw a few simple consequences from what has gone before. We continue to suppose that $\mathcal{K}$ has Eilenberg-Moore objects for monads and comonads and splittings for idempotent 2-cells. 
We shall use several times the following standard result:

Lemma 4.1. For any commutative square

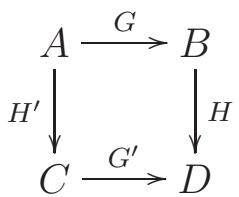

with $H$ and $H^{\prime}$ fully faithful, if $G^{\prime}$ has a right adjoint $R^{\prime}$ and $R^{\prime} H$ lands in $A$, in the sense that $R^{\prime} H \cong H^{\prime} R$ for some $R$, then this $R$ gives a right adjoint to $G$. In particular, if $H$ is fully faithful, and $H G$ has a right adjoint then so does $G$.

Proof. We have natural isomorphisms $B(G a, b) \cong D(H G a, H b)=D\left(G^{\prime} H^{\prime} a, H b\right) \cong$ $C\left(H^{\prime} a, R^{\prime} H b\right) \cong C\left(H^{\prime} a, H^{\prime} R b\right) \cong A(a, R b)$.

First of all, observe that the composite of the embedding $H: \operatorname{Wdl}(\mathcal{K}) \rightarrow \mathcal{K}^{\mathbf{2} \times \mathbf{2}}$ and $Y: \mathcal{K} \rightarrow \operatorname{Wdl}(\mathcal{K})$ is the diagonal 2-functor $\Delta: \mathcal{K} \rightarrow \mathcal{K}^{\mathbf{2} \times \mathbf{2}}$ sending an object $X$ to the square

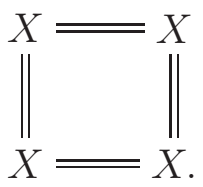

We constructed the embedding $H$ using the existence of Eilenberg-Moore objects, but conversely, from the existence of a fully faithful $H: \operatorname{Wdl}(\mathcal{K}) \rightarrow \mathcal{K}^{\mathbf{2} \times \mathbf{2}}$ satisfying $H Y=\Delta$, we may deduce the existence of a right adjoint to $Y$ by Lemma 4.1, for $\Delta$ certainly has a right adjoint, sending a square to domain of the diagonal (the top left corner in the way we have been drawing squares).

Proposition 4.2. There is a fully faithful 2-functor $J: C \operatorname{md}(\mathcal{K}) \rightarrow \operatorname{Wdl}(\mathcal{K})$ sending a comonad $(K, c)$ to $(K, 1, c, 1)$, and this 2-functor has a right 2-adjoint.

Proof. Here we use the embedding $H_{c}: \operatorname{Cmd}(\mathcal{K}) \rightarrow \mathcal{K}^{2}$ sending a comonad $(K, c)$ to the forgetful $v: K^{c} \rightarrow K$. We also use the fully faithful map $J^{\prime}: \mathcal{K}^{\mathbf{2}} \rightarrow \mathcal{K}^{\mathbf{2} \times \mathbf{2}}$ sending a morphism $v: M \rightarrow K$ to the square

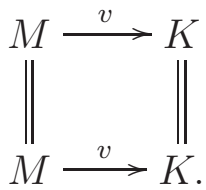

The composite $J^{\prime} H_{c}$ clearly lands in the image of $H$, and so we obtain a fully faithful $J$ with $H J=J^{\prime} H_{c}$. Now $J^{\prime}$ has a right adjoint sending a square

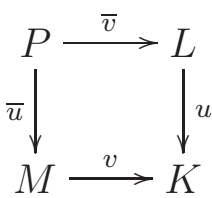

to $\bar{v}: P \rightarrow L$, and this map is comonadic if the original square is in the image of $\operatorname{Wdl}(\mathcal{K})$, thus we obtain by Lemma 4.1 the desired right adjoint.

Dually we have 
Proposition 4.3. There is a fully faithful 2-functor $J_{*}: \operatorname{Mnd}(\mathcal{K}) \rightarrow \operatorname{Wdl}(\mathcal{K})$ sending a monad $(K, t)$ to $(K, t, 1,1)$, and this 2-functor has a right 2-adjoint.

Finally we observe that the diagram

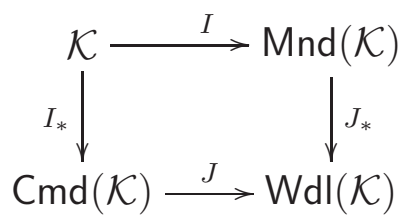

of fully faithful 2-functors commutes, with the diagonal being what we have called $Y$. Thus the corresponding diagram of right adjoints commutes up to natural isomorphism.

\section{REFERENCES}

[1] J. Beck, Distributive laws, [in:] Seminar on Triples and Categorical Homology Theory, B. Eckmann (ed.), Springer LNM 80, 119-140 (1969).

[2] G. Böhm, Doi-Hopf modules over weak Hopf algebras, Comm. Algebra 28 (2000), no. 10, 4687-4698.

[3] G. Böhm, The weak theory of monads, Adv. in Math. 225 (2010), 1-32.

[4] G. Böhm, F. Nill and K. Szlachányi, Weak Hopf algebras I: Integral theory and $C^{*}$ structure, J. Algebra 221 (1999), 385-438.

[5] T. Brzeziński, The structure of corings. Induction functors, Maschke-type theorem, and Frobenius and Galois-type properties, Algebr. and Representat. Theory 5 (2002), 389-410.

[6] T. Brzeziński and S. Majid, Coalgebra bundles, Comm. Math. Phys. 191 (1998), 467492.

[7] T. Brzeziński, R.B. Turner and A.P. Wrightson, The structure of weak coalgebraGalois extensions, Comm. Algebra 34 (2006), no. 4, 1489-1519.

[8] S. Caenepeel and E. De Groot, Modules over weak entwining structures, [in:] New Trends in Hopf Algebra Theory, N. Andruskiewitsch, W.R. Ferrer Santos and H-J. Schneider (eds.), Contemp. Math. 267, 4701-4735, AMS Providence 2000.

[9] S. Caenepeel and E. De Groot, Galois theory for weak Hopf algebras, Rev. Roumaine Math. Pures Appl. 52 (2007), 51-76.

[10] G.M. Kelly and R. Street, Review of the elements of 2-categories, [in:] Category Sem. Proc. Sydney 1972/1973, G.M. Kelly (ed.) Springer LNM 420, 75-103 (1974).

[11] S. Lack, Welcome to weak world. Talk given at the conference CT2009 in Cape Town in July 2009, based on joint work with G. Böhm and R. Street.

[12] J. Power and H. Watanabe, Combining a monad and a comonad, Theoret. Comput. Sci. 280 (2002), 137-162.

[13] R. Street, The formal theory of monads, J. Pure and Applied Algebra 2 (1972), 149168.

[14] R. Street, Weak distributive laws, Theory and Applications of Categories 22 (2009), 313-320. 
Research Institute for Particle and Nuclear Physics, Budapest, H-1525 Budapest 114, P.O.B. 49, Hungary

E-mail address: G.Bohm@rmki.kfki.hu

School of Computing and Mathematics University of Western Sydney, Locked Bag 1797 Penrith South DC NSW 1797, Australia And

Mathematics Department Macquarie University, NSW 2109 Australia.

E-mail address: s.lack@uws.edu.au; steve.lack@mq.edu.au

Mathematics Department Macquarie University, NSW 2109 Australia.

E-mail address: ross.street@mq.edu.au 